\title{
La production du schwa par des apprenants suédophones de FLE
}

\author{
Monika Stridfeldt (L’Université de Dalarna, Suède)
}

\begin{abstract}
The purpose of this paper is to examine the pronunciation of French schwa by Swedish learners of French as a foreign language. The study investigates how the learners deal with schwa deletion (mute e), which is a very frequent phonological process in spoken French, and also how the learners pronounce the schwa when it is not deleted.

Thirty learners participated in the first part of the study. Their task was to read and repeat isolated terms from two lists of words and to read a short text. The second part of the study consists of dialogues between ten learners.

The results show that many learners have difficulties in pronouncing French schwa correctly. In fact, they often pronounce it as $[\mathrm{e}]$ or $[\varepsilon]$ (e.g. revanche

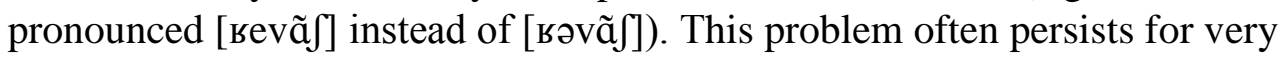
advanced learners, even though learners who have spent at least six months in a French-speaking country generally have better results than those who have spent less time in the target culture. However, there is a wide variation among learners and also from one word to another. As to schwa deletion, the results show that the learners delete very few schwas. They make more schwa deletions in conversation than when they read a text, but compared to native French speakers (Lyche 2016), the learners make considerably fewer schwa deletions. Moreover, the learners make very few incorrect schwa deletions, unlike what has been observed among Dutch learners (Nouveau et Detey 2007).
\end{abstract}

Keywords: L2 acquisition, Swedish learners, L2 French, schwa, phonological variation.

\section{Introduction}

Le schwa, appelé également $e$ caduc, e muet ou $e$ instable, est l'un des processus phonologiques caractéristiques du français. Il s'agit d'une voyelle non accentuée qui correspond très souvent à un <e> graphique et dont la particularité est qu'elle peut s'effacer en fonction d'un certain nombre de facteurs. L'effacement ou le maintien du schwa dépend de facteurs contextuels tels que la position de la syllabe, le nombre et le type de consonnes qui l'entourent, mais aussi de la fréquence du mot et de facteurs socio-situationnels comme le débit, le registre et l'origine géographique du locuteur, etc. Le système cible, pour les apprenants aussi bien que dans notre présentation du schwa ici, est le français de référence (voir p.ex. Lyche 2010). Le principe général est que le schwa se maintient s'il est précédé d'au moins deux consonnes et suivi d'au moins une consonne, sinon il peut tomber. Ainsi, le schwa est systématiquement réalisé en syllabe initiale de mot dans la grenade (mais pas dans 
la petite où l'effacement est facultatif), en syllabe interne dans gouvernement (mais pas dans bêtement), en position finale dans notre liberté (mais pas dans notre ami) et dans le monosyllabe le dans avec le père (mais pas dans chez le père où l'effacement est facultatif).

Lyche $(2010,154)$ note que le timbre du schwa est «sujette à une importante variation, alternant entre [ə], [œ] ou [ø]». Bürki, Racine, Andreassen, Fougeron et Frauenfelder (2008) constatent que le timbre du schwa varie selon la variété régionale.

Le schwa, notamment l'alternance entre présence et absence, fait partie des difficultés que rencontrent les apprenants de français langue étrangère (FLE), aussi bien en production qu'en perception. La présente étude vise à examiner l'effacement et le maintien du schwa par des apprenants suédophones, ainsi que la qualité phonétique du schwa lorsqu'il est maintenu. Nous présenterons la problématique dans la section 2. Dans la section 3, nous présenterons notre étude expérimentale et analyserons les données obtenues, ce qui nous permettra de résumer nos conclusions dans la section 4 .

\section{Le schwa pour les apprenants de FLE}

Selon Nouveau et Detey (2007, 88-90), les difficultés liées à la maîtrise du comportement du schwa surgissent à trois niveaux: au niveau de la perception/compréhension, au niveau de l'impact que peuvent avoir les représentations orthographiques et au niveau de la production.

Andreassen et Lyche $(2018,17)$ soulèvent la question de l'input. Elles constatent que les apprenants FLE sont exposés à une langue qui se caractérise entre autres par un rythme lent, ce qui favorise le maintien des schwas dits variables. En outre, à propos de l'influence de la graphie, Andreassen et Lyche signalent que ces apprenants sont dès le départ confrontés au français dans la forme écrite.

En utilisant des tâches de répétition de mot et de décision lexicale, nous avons montré (Stridfeldt 2005) que l'effacement du schwa complique la perception du français parlé pour les apprenants suédophones. Les mots où les schwas sont effacés sont plus difficilement reconnus par les apprenants. Nouveau (2012) a étudié l'impact de l'effacement du schwa sur la reconnaissance lexicale chez des apprenants néerlandophones du FLE. En utilisant une tâche de dictée, elle a trouvé que les apprenants ont des difficultés à détecter les schwas effacés. Dans une autre étude, Nouveau et Detey $(2007,94)$ ont observé, au niveau de la production, que les apprenants néerlandophones débutants prononcent systématiquement tous les schwas finaux. Ils tendent aussi à réaliser les schwas précédés d'une seule consonne dans les syllabes initiales et internes et dans les séries de clitiques, donc des schwas qui tombent en général chez les locuteurs natifs. Or, à un niveau plus avancé, Nouveau et Detey $(2007,96)$ ont observé chez les apprenants néerlandophones une généralisation abusive de l'effacement du schwa, les apprenants faisant tomber des schwas dont la prononciation est systématique.

Parfois, l'effacement du schwa fait disparaître la frontière lexicale en faveur d'une nouvelle frontière syllabique. En effet, Racine et Grosjean (2000) ont montré que lors de l'effacement du schwa dans un groupe de deux mots (déterminant + substantif), la consonne initiale du substantif peut se rattacher soit à gauche, avec le déterminant (ex. la recette prononcé [las.set]), soit à droite, avec la deuxième syllabe du substantif (ex. la pelouse prononcé [la.pluz]). Remarquons que le rattachement à gauche ne respecte pas l'unité du mot : la frontière syllabique prend le pas sur la frontière lexicale. Par contre, dans le cas d'un rattachement à droite, la frontière syllabique coïncide avec la frontière lexicale. Pour cette 
raison, nous faisons l'hypothèse que le rattachement à gauche sera plus difficile pour les apprenants, aussi bien en perception qu'en production. Il est possible que ce décalage des frontières de mots à gauche apparaisse comme quelque chose d'étrange pour les apprenants, d'autant plus s'ils ont commencé à maîtriser l'enchaînement consonantique et la liaison. Contrairement à l'enchaînement et à la liaison, qui provoquent également des resyllabations mais toujours à droite, l'effacement du schwa avec rattachement à gauche ne renforce pas la syllabation ouverte, mais augmente au contraire le nombre de syllabes fermées. Le rattachement à gauche peut effectivement poser problème au niveau de la perception, ce que montrent les résultats de Nouveau $(2012,74)$ pour des apprenants néerlandophones. Des stimuli comme le refuge, prononcé avec effacement du schwa et rattachement à gauche, [Іәь.fy3], ont causé des erreurs d'interprétation lexicale chez les apprenants, qui ont produit des transcriptions comme leur fuge, l'eurfuge, l'heurfuge et l'heurefuge.

Dans une étude menée auprès d'apprenants norvégophones, Andreassen et Lyche (2018) constatent que l'apprenant est loin du système cible quant à la maîtrise de l'alternance du schwa, même à un niveau avancé. Les apprenants de leurs deux corpus de parole conversationnelle effacent très peu de schwas par rapport aux locuteurs francophones natifs.

Outre la difficulté de savoir si un schwa doit être effacé ou maintenu, la prononciation du schwa pose problème aux apprenants au niveau du timbre. En effet, le schwa français n'a pas le même timbre que le schwa utilisé dans beaucoup d'autres langues. A ce propos, Malmberg $(1976,74)$ fait remarquer :

Le [ə] français n'est pas phonétiquement une voyelle neutre (un « schwa ») comme l'élément vocalique correspondant de tant d'autres langues. Il se prononce pleinement ou disparaît complètement. Il n'y a rien d'intermédiaire. Les anglophones et les Allemands doivent bien prendre garde de ne pas identifier le [ə] français avec le [ə] anglais de better /'betə/ ou le [ə] allemand de bitte /'bitə/ qui sont des voyelles moyennes sans labialisation. Il en est de même des $e$ atones des langues scandinaves.

De plus, la lettre <e> fait partie du problème. Comme le relève Racine $(2015,150)$, les apprenants hispanophones prononcent très souvent le schwa français comme $[\varepsilon]$, par exemple dans des mots comme cheval ou le. Comme le souligne Racine (ibid.),

Un grand nombre d'apprenants n'ont généralement pas de difficultés articulatoires à prononcer le schwa français et se montrent tout à fait capables de répéter correctement le mot pour autant qu'on les sensibilise à ce phénomène. En revanche, au moment de le produire à partir de sa forme graphique, la correspondance phonie-graphie pour la lettre <e> est transférée automatiquement de la L1, ce qui donne lieu à des productions de type $\left[\int \varepsilon v a l\right]$ et $[1 \varepsilon]$.

Pour les suédophones, l'articulation du schwa n'est pas difficile, parce que les voyelles [œ] et $[\varnothing]$ font partie du système vocalique suédois, mais en suédois, ces voyelles sont représentées à l'écrit par la lettre <ö> tandis que la lettre <e> ne se prononce jamais [œ] ou $[\varnothing]$, mais $[e]$ ou $[\varepsilon]$.

Andreassen et Lyche (2016) ont trouvé, chez des apprenants norvégophones, que la réalisation du schwa est plus proche de la cible dans les monosyllabes (ex. je) que dans les polysyllabes (ex. semaine), souvent réalisées avec un [e].

En nous appuyant sur les travaux cités ci-dessus, nous faisons les hypothèses suivantes : 
Pour la question de la chute ou le maintien du schwa, nous nous attendons à observer chez nos apprenants suédophones (apprenants avancés) des effacements du schwa dont la prononciation est systématique en français de référence. Néanmoins, nous nous attendons à trouver un taux de chute nettement inférieur à celui observé chez des locuteurs natifs. En outre, nous nous attendons à trouver très peu d'effacements du schwa avec rattachement à gauche. Quant à la question du timbre du schwa lorsqu'il se réalise, nous nous attendons à ce que l'<e graphique influence la prononciation de façon négative, résultant en la prononciation $[\mathrm{e}]$ ou $[\varepsilon]$, surtout en lecture.

\section{Etude expérimentale}

\subsection{Méthode}

Nous avons effectué des enregistrements avec 30 apprenants suédophones dans le cadre du projet Interphonologie du français contemporain, IPFC (Detey et Kawaguchi 2008; Racine, Detey, Zay et Kawaguchi 2012). Toutes les enquêtes du projet IPFC sont réalisées à partir d'un protocole commun. Ce protocole a été adapté à partir de celui du projet Phonologie du français contemporain, PFC (Durand, Laks et Lyche 2002; Durand, Laks et Lyche 2009) et comprend six tâches différentes :

i. La répétition d'une liste spécifique de mots lue par un francophone natif

ii. La lecture de la liste de mots PFC

iii. La lecture de la liste spécifique

iv. La lecture du texte PFC, intitulé « Le Premier Ministre ira-t-il à Beaulieu ? »

v. Un entretien guidé (conversation entre un enseignant et un apprenant)

vi. Un entretien libre (conversation entre deux apprenants)

Nous avons suivi ce protocole pour constituer notre corpus d'apprenants suédophones. Dans la présente étude, nous nous limiterons à l'analyse des données issues de la répétition et de la lecture de la liste spécifique (tâche 1 et 3), de la lecture du texte PFC (tâche 4) et de l'entretien libre (tâche 6).

La liste spécifique utilisée pour la tâche de répétition ainsi que pour la tâche de lecture contient 3 mots avec schwa : revenant (deux schwas), repartir et le pas. Dans le texte PFC, qui a la forme d'un bref article dans un journal régional, nous avons choisi d'étudier 65 occurrences de mots avec schwa.

Trente étudiants de 1ère année de français à l'Université de Dalarna, 8 hommes et 22 femmes, ont participé à notre expérience. Ces apprenants, âgés de 19 à 69 ans, avaient tous le suédois comme langue maternelle. Avant de commencer leurs études universitaires de français, ils avaient fait entre trois et six années d'études de français à l'école secondaire. Dixhuit des apprenants avaient passé au moins une année dans un pays francophone. Les 30 sujets peuvent être considérés comme des apprenants avancés, se situant entre le niveau B1 et B2 du Cadre européen commun de référence pour les langues (CECRL).

La première partie de notre analyse portera sur la lecture et la répétition des mots ainsi que sur la lecture du texte PFC pour tous les 30 apprenants. La deuxième partie de l'analyse traitera des données de l'entretien libre pour 10 locuteurs, choisis aléatoirement parmi les 30 apprenants. 
Nous considérons ici comme schwa toutes les voyelles graphiques <e > de timbre [ə], [œ] ou [ø], qu'elles soient susceptibles de tomber ou non. Par conséquent, nous considérons la voyelle dans la syllabe initiale du mot premier comme un schwa, même si elle se réalise systématiquement en français de référence. Ce choix est motivé par le fait que Nouveau et Detey (2007) ont observé ce type d'effacements chez leurs apprenants avancés néerlandophones. En outre, nous nous intéressons à la qualité phonétique de ces schwas chez les apprenants. Nous ne traitons pas ici les schwas qui sont généralement muets dans les variétés septentrionales. Ainsi, sont exclus de notre étude les schwas finaux de mot précédés d'une voyelle (ex. vie ), les schwas finaux de mot suivis d'une voyelle (ex. Ministre ira; commune $a u$ ), les schwas finaux de mot suivis d'une consonne et précédés d'une seule consonne (ex. cette commune) et les schwas internes de mot précédés d'une seule consonne (ex. normalement $)^{1}$. Nous n'étudions pas non plus ici les schwas finaux précédés d'au moins deux consonnes à gauche et suivis d'au moins une consonne à droite (ex. Ministre ne ; notre liberté), bien que ces schwas soient en général réalisés.

Pour les tâches de répétition et de lecture, nous avons indiqué pour chaque locuteur si les schwas étaient prononcés ou effacés et s'ils étaient prononcés correctement ou non. Nous avons traité comme correctes les prononciations [ə], [œ] et [ø] ainsi que les effacements conformes au français de référence. Pour l'entretien libre, nous avons transcrit 10 minutes de dialogue pour chaque apprenant. Nous avons indiqué pour chaque mot comportant un schwa s'il était maintenu ou effacé et s'il était prononcé correctement ou non.

\subsection{Résultats et discussion}

\subsubsection{Résultats pour la lecture et la répétition de la liste spécifique}

La figure 1 présente le taux de prononciation correcte pour la répétition de mots et pour la lecture des mêmes mots de la liste spécifique. Il s'agit de la moyenne des 30 apprenants pour ces trois mots. Comme revenant contient deux schwas, ce mot apparaît deux fois dans la figure, d'abord pour le premier schwa puis pour le second, ce qui est illustré par un E majuscule. Il convient de signaler que, pour ces trois mots, c'est seulement pour revenant que l'on pourrait s'attendre à l'effacement du schwa, et seulement pour la deuxième syllabe de ce mot, étant donné qu'il s'agit de la lecture d'une liste de mots et que le schwa est en général maintenu en syllabe initiale de groupe rythmique. La voix modèle que les apprenants écoutent dans la tâche de répétition, prononce cependant les deux schwas.

\footnotetext{
${ }^{1}$ Nous avons néanmoins choisi d'inclure le mot indiqueraient parce que le schwa de ce mot a été prononcé par presque tous les apprenants.
} 


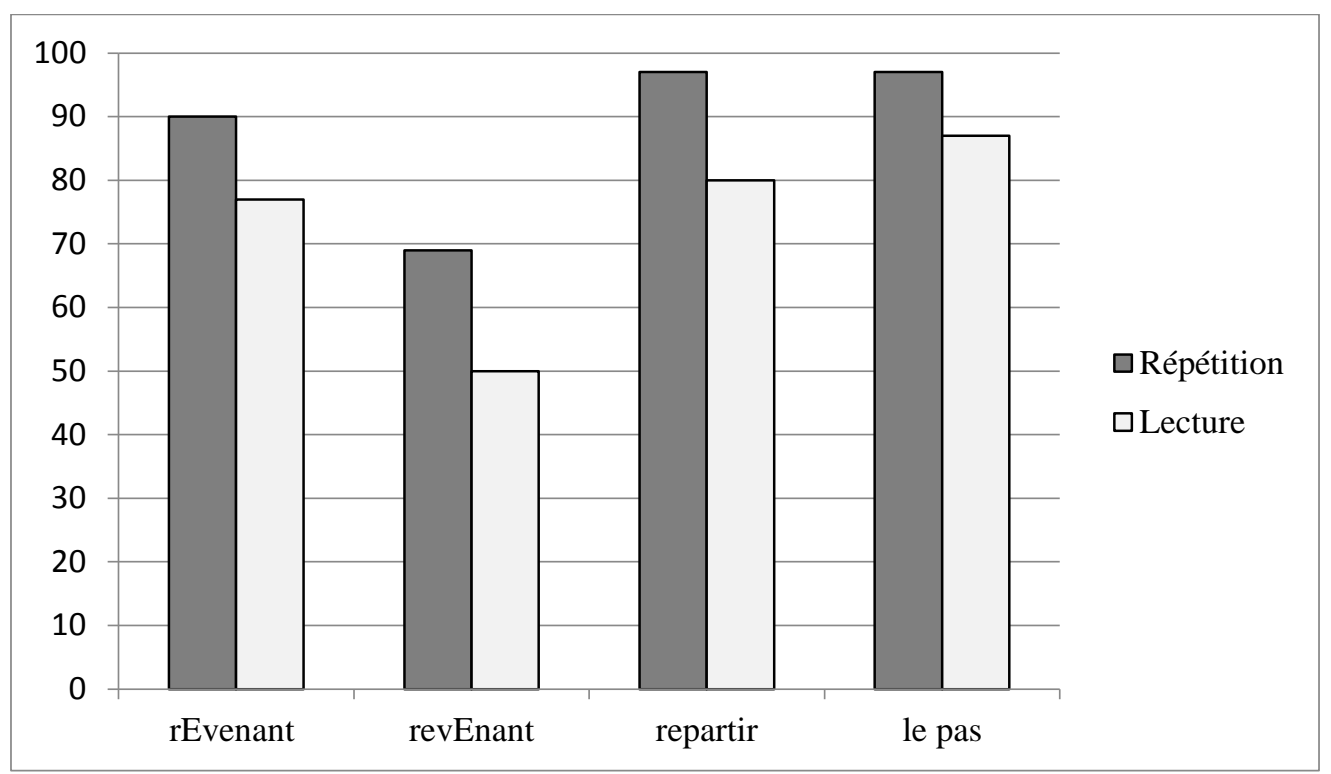

Fig. 1 : Taux de prononciation correcte pour la répétition de mots et pour la lecture des mêmes mots de la liste spécifique.

Nous pouvons constater que les apprenants prononcent des schwas qui sont plus proches de la prononciation cible $[\curvearrowright, \propto, \varnothing]$ lors de la tâche de répétition (taux moyen de prononciation correcte de $88,25 \%$ ) que lors de la lecture (taux moyen de prononciation correcte de $73,5 \%$ ), ce qui n'est pas surprenant étant donné qu'ils écoutent et répètent la prononciation d'une locutrice native. Comme l'a signalé Racine $(2015,150)$, la plupart des apprenants n'ont pas de difficultés articulatoires à prononcer le schwa et se montrent tout à fait capables de le répéter correctement. Nous pouvons en outre constater que le premier schwa de revenant est plus proche de la cible que le deuxième. Il semble que les locuteurs aient fait plus attention à la première syllabe. Sont considérées comme non correctes les prononciations [e] ou [ $[\varepsilon]$ ou un [ə] trop lâche et pas assez arrondi. Comme on pouvait s'y attendre, c'est seulement le schwa de la deuxième syllabe du mot revenant qui chute quelques fois : parmi les $50 \%$ de prononciation correctes, on trouve $13 \%$ d'effacement, [bəvnã], et $37 \%$ de [ə], [œ] ou [ø]. Dans la tâche de répétition, aucun apprenant n'efface ce schwa, comme la voix modèle le prononce.

\subsubsection{Résultats pour la lecture du texte}

Le tableau 1 présente le taux de prononciation correcte pour chaque mot avec les effacements indiqués.

\begin{tabular}{|lrrr|}
\hline Mots & Taux de prononciation correcte & $\begin{array}{l}\text { Nombre } \\
\text { d'occurrences }\end{array}$ & $\begin{array}{l}\text { Variation selon } \\
\text { l'occurrence }\end{array}$ \\
\hline que & $4(\times 30=120)$ & $90 \%$ & $87-93 \%$ \\
\hline ne & $3(\times 30=90)$ & $77 \%$ & $70-87 \%$ \\
\hline$\underline{\text { le }}$ & $13(\times 30=390)$ & $74 \%$ & $47-90 \%$ \\
\hline ce & $3(\times 30=90)$ & $74 \%$ & $70-77 \%$ \\
\hline
\end{tabular}




\begin{tabular}{|c|c|c|c|}
\hline de & $23(x 30=690)$ & $66 \%(65 \%[\partial]+5$ effacements $=1 \%)$ & $48-80 \%$ \\
\hline se & $4(x 30=120)$ & $65 \%$ & $47-80 \%$ \\
\hline Premier & $7(x 30=210)$ & $50 \%$ & $40-59 \%$ \\
\hline chemises & $1(x 30=30)$ & $40 \%(33 \%[\boldsymbol{\partial}]+2$ effacements $=7 \%)$ & \\
\hline quelques & $2(x 30=60)$ & $39 \%$ & $37-40 \%$ \\
\hline revanche & $1(\mathrm{x} 30=30)$ & $27 \%$ & \\
\hline seraient & $1(x 30=30)$ & $20 \%$ & \\
\hline dẹpuis & $1(x 30=30)$ & $17 \%$ & \\
\hline gouvernement & $1(\mathrm{x} 30=30)$ & $17 \%$ & \\
\hline indiqueraient & $1(x 30=30)$ & $10 \%(7 \%[\boldsymbol{\partial}]+1$ effacement $=3 \%)$ & \\
\hline Total: & $65(x 30=1950)$ & 8 effacements au total & \\
\hline
\end{tabular}

Tableau 1 : Taux de prononciation correcte pour chaque mot dans la lecture du texte.

Il ressort du tableau que le mot $d e$ a le plus grand nombre d'occurrences : il y en a 23 dans le texte. Ces 23 occurrences ont été prononcées par les 30 apprenants, ce qui donne 690 occurrences. Parmi ces 690 occurrences, on n'en trouve que 5 prononcées avec effacement du schwa. En moyenne le mot de a été correctement prononcé dans $66 \%$ des cas $(65 \%$ de [ə] et $1 \%$ d'effacement). Le taux de prononciation correcte varie entre 48 et $80 \%$ pour les 23 occurrences. Le contexte gauche ne ressort pas du tableau mais est important pour savoir si la chute du schwa est possible. Parmi les 23 occurrences du mot de, le schwa est précédé d'une seule consonne prononcée $(\mathrm{V}(\#) \mathrm{C} ə)$ dans 10 cas (ex. décidé de), ce qui veut dire que la chute est possible, sauf pour le contexte De plus, situé au début d'une phrase. Le schwa est précédé de deux consonnes prononcées $(\mathrm{C}(\#) \mathrm{C} ə)$ ou plus dans 13 cas (ex. village de), donc un contexte où le schwa est généralement maintenu.

Outre de, c'est seulement pour les mots chemises et indiqueraient qu'on trouve quelques cas d'effacement du schwa (et aussi erronément pour le mot gouvernement): chemises est prononcé avec effacement par 2 apprenants et indiqueraient par un seul apprenant. Il est intéressant de constater que indiqueraient a été prononcé incorrectement

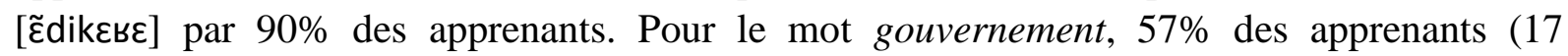
apprenants) font tomber le schwa erronément : [guvesnmã] ${ }^{2}$, peut-être sous l'influence du mot anglais government.

Pour les 1950 occurrences de mots (65 occurrences x 30 apprenants), seulement 8 ont été prononcés avec effacement, ce qui correspond à $0,4 \%$. Nous pouvons donc constater que les apprenants ont effacé très peu de schwas en lisant ce texte. Regardons de plus près les contextes dans lesquels se trouvent ces schwas effacés. La séquence tournée d(e) la région ${ }^{3}$ a été produite avec effacement par deux apprenants, la séquence pas d(e) la réaction également par deux apprenants et la séquence membre d(e) l'opposition par un apprenant. La suite ses ch(e)mises en soie a été prononcée avec effacement par deux apprenants. Le mot

\footnotetext{
${ }^{2}$ Toutefois, selon Durand et Eychenne (2004, 326), l'effacement du schwa dans gouvernement est possible dans certaines variétés et sous certaines conditions.

${ }^{3}$ Nous avons mis les schwas effacés entre parenthèses ici.
} 
indiqueraient, qui a été prononcé avec effacement par un étudiant, figure dans la séquence $L e$ Nouvel Observateur indiqu(e)raient que des activistes... Il est à noter que les effacements faits par les apprenants sont des effacements avec rattachement à droite : de la [dla], de l'opposition [dlo.po.zi.sjõ] et chemises [ $\mathrm{Jmiz}]$. Or, le texte contient quelques occurrences potentielles d'effacement avec rattachement à gauche, par exemple détachement $d(e)$ police

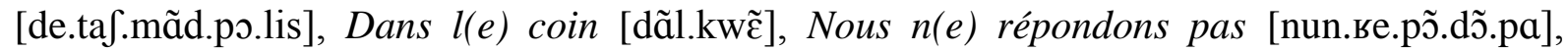
Nous avons l(e) soutien [nu.za.võl.su.tjẽ] et $I l$ a l(e) sentiment [i.lal.sã.ti.mã]. Aucun des apprenants n'a fait tomber le schwa dans ces phrases et n'a donc produit aucun rattachement à gauche, ce qui renforce notre hypothèse que le rattachement à gauche est plus difficile à maîtriser pour les apprenants.

Pour ce qui est de la qualité acoustique du schwa, il est intéressant d'observer que les monosyllabes, c'est-à-dire de, $l e, s e$, que, ne et $c e$ sont plus proches de la cible que les mots à plusieurs syllabes, ce qui rappelle les résultats d'Andreassen et Lyche (2016) pour des apprenants norvégophones. La raison de cela pourrait être que ces mots sont très fréquents et bien connus par les apprenants. Le mot quelques devrait également être bien connu, mais il est possible que le $[\varepsilon]$ de la première syllabe engendre la prononciation erronée $[\mathrm{k} \varepsilon l \mathrm{k} \varepsilon]$.

La figure 2 présente le taux de prononciation correcte ( $[ə, \propto, \varnothing]$ ou effacement) pour chaque locuteur pour la lecture du texte. L'effacement du schwa est marqué en gris clair. Sont considérées comme non correctes les prononciations [e] ou $[\varepsilon]$ ou un [ə] trop lâche et pas assez arrondi.

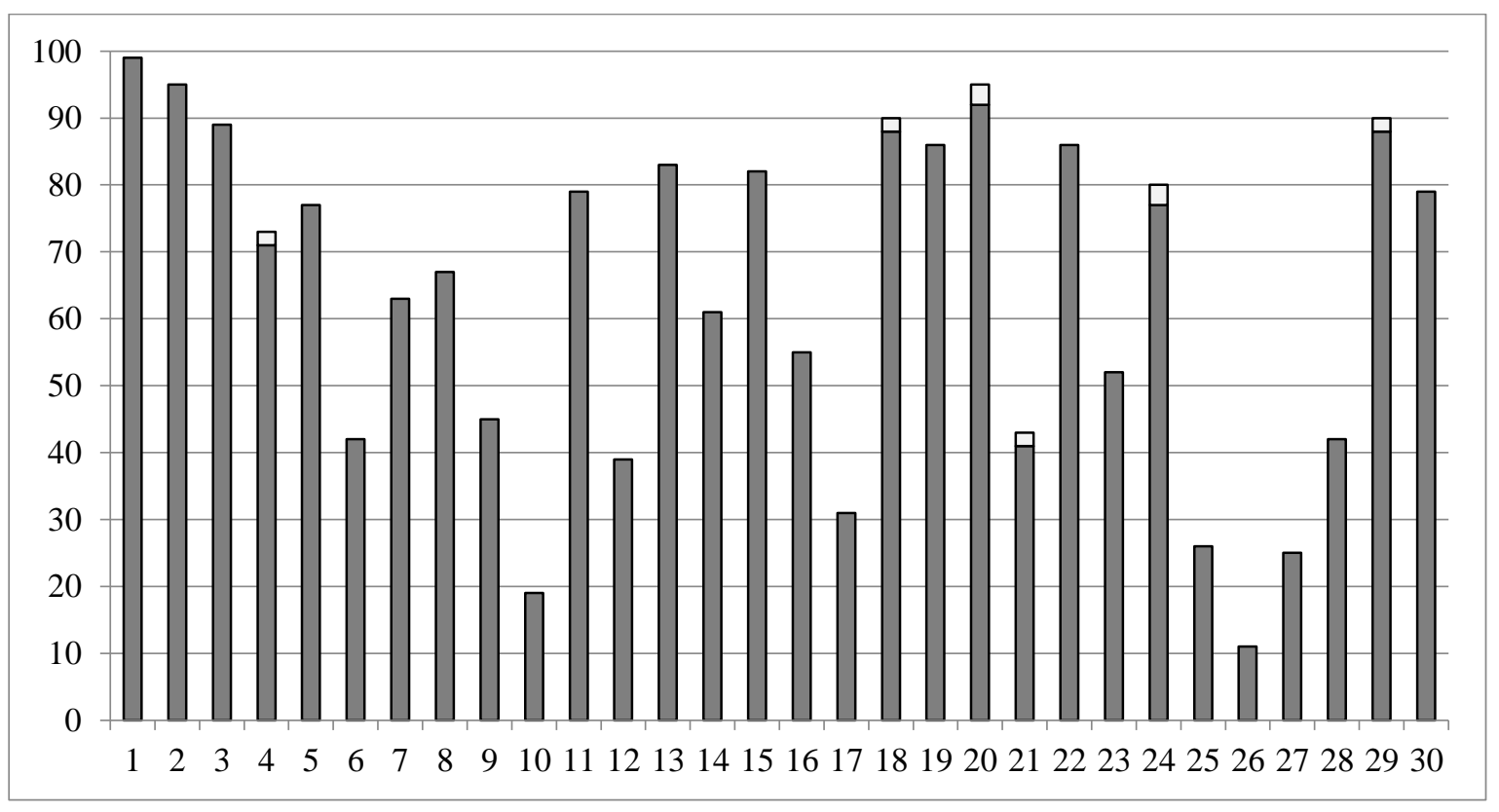

Fig. 2 : Taux de prononciation correcte pour chaque locuteur dans la lecture du texte.

On trouve une grande variation entre les différents apprenants. Il ressort de la figure 2 que l'apprenant numéro 1 a prononcé 99\% des schwas correctement tandis que l'apprenant 26 n'en a produit que $11 \%$ correctement. Onze des 30 apprenants, soit un tiers, prononcent au moins $80 \%$ des schwas correctement. Dix apprenants, également un tiers, prononcent moins de la moitié des schwas correctement. En ce qui concerne l'effacement du schwa, on en trouve chez seulement six des 30 apprenants. Il est intéressant de noter que l'apprenant 1 qui a prononcé le plus de schwas correctement n'a produit aucun effacement. La capacité de 
réaliser des schwas dont le timbre est conforme à la prononciation cible n'implique donc pas nécessairement la maîtrise de l'alternance du schwa. Toutefois, des études effectuées avec des locuteurs natifs francophones dans le cadre du projet PFC ont montré que le taux d'effacement est assez bas en lecture. Selon Lyche (2016, 362), le taux d'effacement en lecture est $8,62 \%$ pour les monosyllabes et $1,47 \%$ pour les syllabes initiales de polysyllabes. Ces résultats sont issus de 13 points d'enquête de la partie nord de la France.

\subsubsection{Résultats pour la parole conversationnelle}

La figure 3 présente une comparaison entre les tâches de lecture et de conversation libre. La figure montre le taux de prononciation correcte (réalisation conforme à la prononciation cible) pour chacun des 10 apprenants pour la lecture des mots du texte (bâton gauche) et pour la parole conversationnelle (bâton droit). Les effacements du schwa sont marqués en gris clair. Ce qui est marqué en gris foncé correspond par conséquent à la prononciation [ə], [œ] ou [ø]. Sont considérées comme non correctes les prononciations [e]

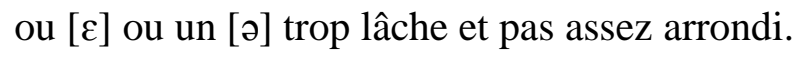

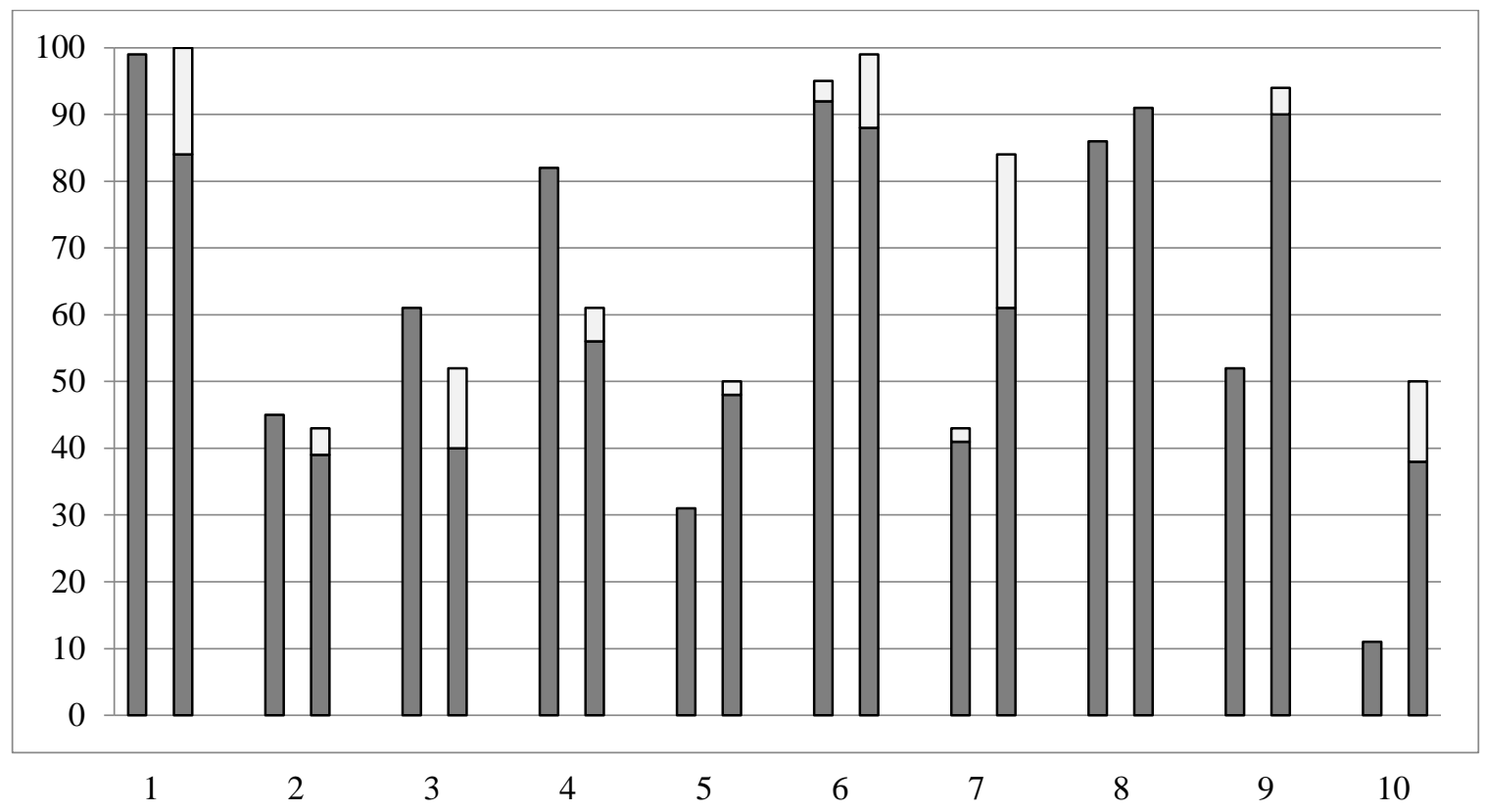

Fig. 3 : Taux de prononciation correcte pour chacun des 10 apprenants pour la lecture des mots du texte (bâton gauche) et pour la parole conversationnelle (bâton droit).

Nous pouvons constater que les apprenants effacent plus de schwas en conversation qu'en lecture du texte, ce à quoi on pouvait s'attendre étant donné qu'il a été montré que les locuteurs natifs maintiennent beaucoup plus de schwas dans une situation de lecture que dans la parole spontanée (voir par exemple Hansen 2000 et Lyche 2016). Dans la tâche de lecture, deux des dix apprenants seulement ont fait quelques effacements, tandis que dans la tâche de conversation, neuf des dix apprenants en ont fait.

En ce qui concerne le timbre, la moitié des étudiants réalisent la plupart des schwas conformément à la cible dans la parole conversationnelle (les étudiants 1, 6, 7, 8 et 9). L'autre moitié ont plus de problèmes (les étudiants $2,3,4,5$ et 10). Ils prononcent le schwa comme [e] ou [ع] ou bien comme un [ə] trop lâche et pas assez arrondi. 
Il ressort de la figure que quatre étudiants ont à peu près les mêmes taux de prononciation correcte pour la lecture du texte et pour la parole conversationnelle, soit les étudiants 1, 2, 6 et 8. Quatre étudiants réalisent des schwas plus proches de la cible dans la conversation que dans le texte lu : les étudiants 5, 7, 9 et 10. Ces résultats, même s'ils ne sont pas très nets, pourraient renforcer l'hypothèse selon laquelle l'<e> graphique influence la prononciation de façon négative. Cependant, pour deux étudiants (numéros 3 et 4), la prononciation est un peu plus loin de la cible dans la conversation.

Les étudiants ayant réussi le mieux au niveau de la qualité phonétique, c'est-à-dire numéro 1, 6 et 8 ont passé un an ou plus dans un pays francophone. L'étudiant 2, dont la réalisation du schwa est loin de la prononciation cible, a passé trois semaines seulement dans un pays francophone. Cependant, l'étudiant 5, dont la prononciation du schwa est également loin de la cible, vit en France depuis 3 ans. Séjourner longuement dans un pays francophone ne garantit donc pas une bonne prononciation du schwa. Un autre exemple est l'étudiant 10 qui a passé deux années en France mais qui ne prononce que $11 \%$ des schwas correctement dans la tâche de lecture et $52 \%$ dans la conversation (ce qui est cependant beaucoup mieux).

Il peut être intéressant de regarder la variation de réalisation pour les différents mots. Le tableau 2 présente le taux de prononciation correcte pour les mots qui ont au moins 5 occurrences dans la parole conversationnelle.

\begin{tabular}{|c|c|c|c|}
\hline Mots & $\begin{array}{l}\text { Nombre } \\
\text { d'occurrences }\end{array}$ & $\begin{array}{l}\text { Nombre de prononciation } \\
\text { correcte }\end{array}$ & $\begin{array}{l}\text { Taux de prononciation } \\
\text { correcte }\end{array}$ \\
\hline petit & 14 & 14 (dont 4 effacements) & 100 \\
\hline je & 90 & 83 (dont 27 effacements) & 92 \\
\hline que & 89 & 81 & 91 \\
\hline quelques & 10 & 9 (dont 1 effacement) & 90 \\
\hline ce & 17 & 15 (dont 7 effacements) & 88 \\
\hline se & 15 & 13 & 87 \\
\hline me & 11 & 9 (dont 2 effacements) & 82 \\
\hline le & 101 & 82 (dont 5 effacements) & 81 \\
\hline de & 119 & 86 (dont 3 effacements) & 72 \\
\hline ne & 9 & 6 (dont 1 effacement) & 67 \\
\hline premier & 6 & 4 & 67 \\
\hline semaine & 5 & 1 & 20 \\
\hline demi & 6 & 0 & 0 \\
\hline mercredi & 5 & 0 & 0 \\
\hline Total: & 537 & 403 (dont 50 effacements) & 75 \\
\hline
\end{tabular}

Tableau 2 : Taux de prononciation correcte pour les mots qui ont au moins 5 occurrences chez les 10 apprenants de la parole conversationnelle. 
Il ressort du tableau 2 qu'il y a 90 occurrences du mot je et que 83 de ces occurrences sont prononcées correctement. Parmi ces 83 occurrences correctes il y a 27 effacements du schwa. Comme pour la lecture du texte, les mots monosyllabiques sont prononcés correctement la plupart du temps. Ils ont un taux de prononciation correcte entre $67 \%$ pour ne et $92 \%$ pour je. La variation est plus grande pour les mots polysyllabiques : petit a toujours été prononcé correctement, tandis que demi et mercredi ont $0 \%$ de prononciation correcte ces mots ont été prononcés avec [e] ou $[\varepsilon]$. En moyenne, les schwas de mots monosyllabiques ont été prononcés correctement dans $83 \%$ des cas tandis que les schwas en position initiale de mots polysyllabiques ont été prononcés correctement dans $56 \%$ des cas. Andreassen et Lyche (2016) avaient trouvé une meilleure qualité du schwa dans les monosyllabes que dans les polysyllabes chez les apprenants norvégiens (qualité [e] en polysyllabes). On peut donc voir la même tendance chez les apprenants suédois.

Il est intéressant de constater qu'il n'y a aucun effacement pour demi. Ce mot figure dans les contextes suivants : neuf demi-journées, une demi-journée, trois heures et demie, trois ans et demi, huit et demi, deux et demi. On aurait pu s'attendre à l'effacement dans les quatre derniers contextes. Le mot semaine a été prononcé correctement une fois et incorrectement [semen] cinq fois, jamais avec effacement. Ce mot apparaît dans les contextes suivants : la semaine, par semaine ( 2 fois), chaque semaine, onze semaines.

C'est normal de ne pas trouver d'effacement du schwa pour les mots premier et mercredi dont la réalisation du schwa est systématique selon les règles générales. Les prononciations erronées du schwa de ces mots sont $[\mathrm{e}]$ ou $[\varepsilon]$.

Nous allons regarder de plus près les effacements du schwa. Au total, les 10 apprenants ont fait 50 effacements : 45 effacements d'un schwa de monosyllabe et 5 effacements d'un schwa initial de mot polysyllabique. Tous ces effacements sont présentés dans le tableau 3.

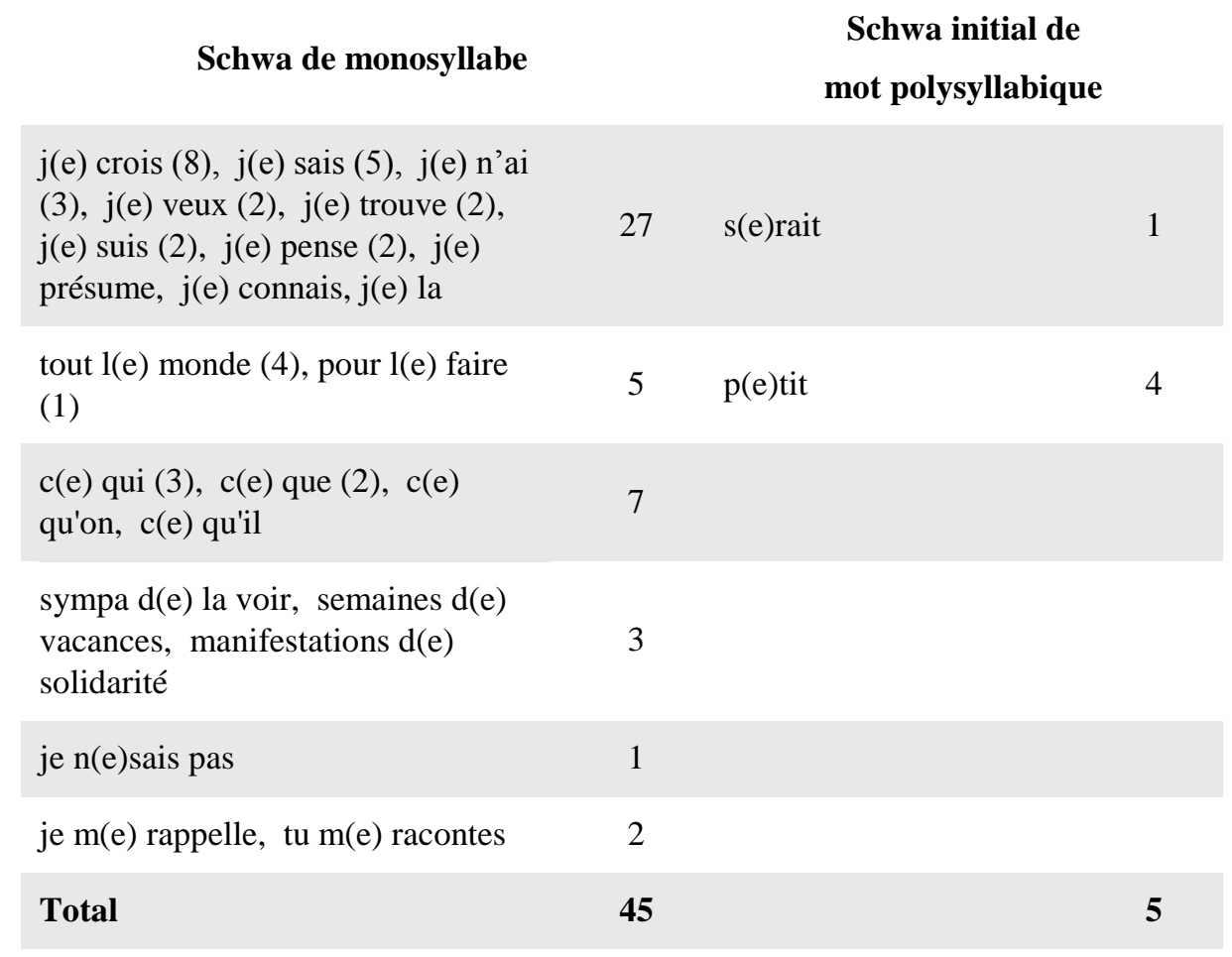

Tableau 3 : Toutes les 50 occurrences d'effacement du [ə] dans la parole conversationnelle des 10 apprenants. 
Il est important de souligner que tous les effacements sont corrects, ce qui n'était pas le cas pour les apprenants néerlandophones (Nouveau et Detey 2007). Nous pouvons constater que la plupart des effacements du schwa ont été effectués dans le clitique je. Andreassen et Lyche $(2018,21)$ ont également observé beaucoup d'effacements dans je chez des apprenants norvégophones, ce qu'elles expliquent par le fait qu'il s'agit d'un mot très fréquent et que la fricative facilite l'articulation. Les 7 effacements effectués dans le mot ce produisent le groupe consonantique /sk/, qui ne pose pas de problème articulatoire pour les suédophones. Le mot polysyllabique serait a été prononcé avec effacement une fois : Ça $s(e)$ rait bien. Le mot petit a été prononcé avec effacement 4 fois sur $14:$ une p(e)tite ferme, un p(e)tit tour, beaucoup de euh... de p(e)tits euh..., un p(e)tit bistro.

La plupart des effacements ont été réalisés avec rattachement à droite, mais on en trouve quelques-uns avec rattachement à gauche, soit tout l(e) monde (4 fois), pour l(e) faire, je $n(e)$ sais pas, je $m(e)$ rappelle et $t u m(e)$ racontes. L'effacement du schwa dans la locution tout l(e) monde était fréquent aussi chez les norvégophones (Andreassen et Lyche 2018, 20).

Le tableau 4 présente les chiffres relatifs pour le maintien et la chute du schwa dans les monosyllabes et dans les syllabes initiales de polysyllabes dans la parole conversationnelle.

\begin{tabular}{|c|c|c|c|c|c|c|c|c|}
\hline \multirow{3}{*}{$\begin{array}{c}\text { Nombre } \\
\text { total }\end{array}$} & \multicolumn{4}{|c|}{ Schwa de monosyllabe } & \multicolumn{4}{|c|}{ Schwa initial de mot polysyllabique } \\
\hline & \multicolumn{2}{|c|}{ Maintien } & \multicolumn{2}{|c|}{ Chute } & \multicolumn{2}{|c|}{ Maintien } & \multicolumn{2}{|c|}{ Chute } \\
\hline & Nombre & Taux & Nombre & Taux & Nombre & Taux & Nombre & Taux \\
\hline 514 & 408 & $90 \%$ & 45 & $10 \%$ & 56 & $92 \%$ & 5 & $8 \%$ \\
\hline
\end{tabular}

Tableau 4 : Nombre et taux de maintien ou chute du schwa en position de monosyllabe et en position initiale de polysyllabe.

Il ressort du tableau que les apprenants ont fait tomber le schwa dans $10 \%$ des monosyllabes et dans $8 \%$ des polysyllabes. Il convient de comparer ces pourcentages à ceux obtenus avec des locuteurs natifs. Sur la base des données PFC de 13 points d'enquête dans la partie nord de la France, Lyche $(2016,362)$ présente des taux de chute du schwa pour les monosyllabes et la syllabe initiale de polysyllabe. En conversation, le taux de chute est de $65,39 \%$ dans les monosyllabes et de $65,82 \%$ dans la syllabe initiale de polysyllabe. Ces données mettent en évidence une très grande différence entre les apprenants et les francophones natifs. Nos apprenants suédophones maintiennent beaucoup plus de schwas que les locuteurs natifs.

Nos résultats ressemblent à ceux d'Andreassen et Lyche (2018), qui ont observé chez des apprenants norvégophones (8 locuteurs, niveau B1/B2) un taux de chute de $10 \%$ dans les monosyllabes et de $11 \%$ dans la syllabe initiale de polysyllabe. Avec un groupe d'apprenants moins avancés (8 locuteurs, niveau A2), Andreassen et Lyche (2018) ont trouvé un taux de chute encore plus bas, avec chute dans $2 \%$ des monosyllabes et dans $1 \%$ des polysyllabes. Il est intéressant de noter qu'il y a une progression entre les groupes en ce que les apprenants avancés maîtrisent mieux l'alternance du schwa, même si, comme le soulignent Andreassen et Lyche $(2018,20)$, ils sont loin du système cible.

\section{Conclusion}

Dans ce travail, nous avons examiné la production du schwa chez des apprenants suédophones. Notre étude a porté à la fois sur la variation maintien/effacement du schwa et sur la manière dont le schwa est prononcé lorsqu'il est réalisé. 
Nous avons constaté que les apprenants effacent très peu de schwas. Il y a, cependant, des différences selon le registre. Ils réalisent presque tous les schwas non finaux en lecture du texte, tandis qu'ils font tomber plus de schwas en conversation, soit $10 \%$ des schwas de monosyllabe et $8 \%$ des schwas initiaux de mot polysyllabique. Toutefois, ces taux de chute sont considérablement inférieurs à ceux observés chez des locuteurs natifs. Nos résultats ressemblent cependant à ceux des apprenants avancés norvégophones de l'étude d'Andreassen et Lyche (2018).

A la différence des apprenants néerlandophones (Nouveau et Detey 2007), nos apprenants suédophones n'ont pas réalisé beaucoup d'effacements du schwa dont la prononciation est systématique en français de référence. Les seuls effacements de ce type ont été observés pour le mot gouvernement qui a été prononcé sans schwa par plus de la moitié des apprenants.

Conformément à notre hypothèse, les apprenants ont produit très peu d'effacements du schwa avec rattachement à gauche (aucun en lecture et 8 en conversation).

En ce qui concerne la qualité acoustique du schwa, elle est plus proche de la cible dans la tâche de répétition de mots que dans celle de lecture de mots. Dans la lecture du texte, un grand nombre d'apprenants ont des problèmes à prononcer le schwa correctement. Ils le prononcent souvent $[\mathrm{e}]$ ou $[\varepsilon]$ ou bien comme une voyelle semblable à [ə] mais moins arrondie. On trouve cependant une grande variation entre les apprenants. Dans la parole conversationnelle, la moitié des 10 apprenants prononcent la plupart des schwas correctement. Ceux-ci ont séjourné un an ou plus dans un pays francophone. L'autre moitié ont plus de difficultés - pour eux c'est la prononciation $[\mathrm{e}]$ ou $[\varepsilon]$ qui prédomine. Deux de ces apprenants ont séjourné moins d'un mois dans un pays francophone, mais les trois autres ont séjourné un an et demi ou plus en milieu francophone. La difficulté concernant le timbre du schwa peut donc persister très longtemps. Comme le fait remarquer Racine (2015, 150), les

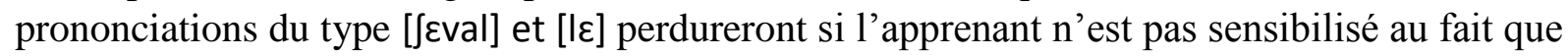
la lettre $<\mathrm{e}>$ possède plusieurs réalisations possibles.

Nous nous étions attendus à ce que les apprenants prononcent des schwas plus proches de la cible en conversation qu'en lecture quand ils n'ont pas devant les yeux les <e> graphiques, et les résultats vont dans ce sens, même s'ils ne sont pas tout à fait convaincants.

La prononciation du schwa est en général plus proche de la cible dans les mots monosyllabiques que dans la syllabe initiale de mots polysyllabiques. Cela pourrait peut-être indiquer que les apprenants ont appris la prononciation correcte des mots courts et très fréquents, tandis qu'ils sont plus influencés par l'écrit pour les mots plus longs et moins fréquents, même en parole conversationnelle.

\section{Bibliographie}

Andreassen, Helene N. et Lyche, Chantal. 2016. Le schwa dans la parole conversationnelle d'apprenants et l'impact des facteurs phonologiques : un début d'analyse. Papier présenté aux Rencontres FLOraL-IPFC 2016, Paris.

Andreassen, Helene N. et Lyche, Chantal. 2018. « Le rôle de la variation dans le développement phonologique : Acquisition du schwa illustrée par deux corpus d'apprenants norvégiens ». In Synergies Pays Scandinaves 13, 13-24.

Bürki Audrey, Racine, Isabelle, Andreassen, Helene. N., Fougeron, Cécile et Frauenfelder, Ulrich, H. 2008. «Timbre du schwa en français et variation régionale : une étude comparative ». In Actes des XXVIIèmes Journées d'études sur la parole, 14, Avignon. 
Detey, Sylvain, Kawaguchi, Yuji. 2008. «Interphonologie du Français Contemporain (IPFC) : récolte automatisée des données et apprenants japonais ». In Journées PFC : Phonologie du français contemporain: variation, interfaces, cognition, Paris.

Durand, Jacques et Eychenne, Julien. 2004. «Le schwa en français. Pourquoi des corpus ?». In Corpus 3, 311-356.

Durand, Jacques, Laks, Bernard et Lyche, Chantal. 2002. "La phonologie du français contemporain: usages, variétés et structure ». In Romanistische Korpuslinguistik Korpora und gesprochene Sprache, éd. Claus Pusch et Wolfgang Raible, 93-106. Tübingen: Gunter Narr Verlag.

Durand, Jacques, Laks, Bernard et Lyche, Chantal. 2009. « Le projet PFC: une source de données primaires structurées ». In Phonologie, variation et accents du français, éd. Jacques Durand, Bernard Laks and Chantal Lyche, 19-61. Paris: Hermès.

Hansen, Anita Berit. 2000. «Le E caduc interconsonantique en tant que variable sociolinguistique: une étude en région parisienne. ». In Linx 42, 45-58.

Lyche, Chantal. 2010. «Le français de référence : éléments de synthèse ». In Les variétés du français parlé dans l'espace francophone : ressources pour l'enseignement, éd. Sylvain Detey, Jacques Durand, Bernard Laks et Chantal Lyche, 143-165. Paris: Ophrys, 2010.

Lyche, Chantal. 2016. "Approaching variation in PFC: the schwa level". In Varieties of spoken French, éd. Sylvain Detey, Jacques Durand, Bernard Laks et Chantal Lyche, 352-362. Oxford: Oxford University Press, 2016.

Malmberg, Bertil. 1976. Phonétique française. Malmö: LiberLäromedel.

Nouveau, Dominique. 2012. "Limites perceptives de l'e caduc chez des apprenants néerlandophones ». In Revue canadienne de linguistique appliquée 15, 1, 60-78.

Nouveau, Dominique et Detey, Sylvain. 2007. «Enseignement/apprentissage du schwa et apprenants néerlandais : des données de la base PFC à l'espace-ressources en ligne du projet PFC-EF ». In Bulletin PFC, 7, 87-106.

Racine, Isabelle. 2015. «La liaison chez les apprenants hispanophones avancés de FLE ». In Bulletin VALS-ASLA, 102, 147-167.

Racine, Isabelle et Grosjean, François. 2000. «Influence de l'effacement du schwa sur la reconnaissance des mots en parole continue » In L'Année psychologique 100/2000, 393-417.

Racine, Isabelle, Detey, Sylvain, Zay, Françoise et Kawaguchi, Yuji. 2012. " Des atouts d'un corpus multitâches pour l'étude de la phonologie en L2: l'exemple du projet Interphonologie du Français Contemporain (IPFC) ». In Recherches récentes en FLE, éd. Alain Kamber et Carine Skupien, 1-19. Bern: Peter Lang.

Stridfeldt, Monika. 2005. La perception du français oral par des apprenants suédois. Thèse de doctorat. Université d'Umeå. 